\title{
The Pathophysiology of Dystonic Tremors and Comparison With Essential Tremor
}

\author{
Pattamon Panyakaew, ${ }^{1,2}$ Hyun Joo Cho, ${ }^{1}$ Sang Wook Lee, ${ }^{3,4,5}$ Tianxia $\mathrm{Wu},{ }^{6}$ and ${ }^{\circledR}$ Mark Hallett ${ }^{1}$ \\ ${ }^{1}$ Human Motor Control Section, National Institute of Neurological Disorders and Stroke, National Institutes of Health, Bethesda, Maryland \\ 20892-1428, ${ }^{2}$ Chulalongkorn Center of Excellence for Parkinson's Disease \& Related Disorders, Department of Medicine, Faculty of Medicine, \\ Chulalongkorn University and King Chulalongkorn Memorial Hospital, Thai Red Cross Society, Bangkok, 10330, Thailand, ${ }^{3}$ Department of \\ Biomedical Engineering, Catholic University of America, Washington, DC 20064, ${ }^{4}$ Center for Applied Biomechanics and Rehabilitation Research, \\ MedStar National Rehabilitation Hospital, Washington, DC 20010, ${ }^{5}$ Department of Mechanical Engineering, Korea Advanced Institute of Science \\ and Technology, Daejeon, 34141, Korea, and ${ }^{6}$ Clinical Neurosciences Program, National Institute of Neurological Disorders and Stroke, National \\ Institutes of Health, Bethesda, Maryland 20892-1428
}

There are two types of dystonic tremor syndromes (DTS), dystonic tremor (DT) and tremor associated with dystonia (TAWD), and neither is understood. DTS likely share some mechanisms with nontremulous dystonia, and there may also be overlaps with essential tremor (ET). We studied 21 ET (8 females, 13 males) and 22 DTS human patients (10 females, 12 males), including 13 human patients with DT (writer's cramp with writing tremor) and 9 human patients with tremor associated with dystonia (TAWD; cervical dystonia with hand tremor). Tremors were analyzed using accelerometry and surface EMG of the antagonist pairs of arm muscles during posture, simple kinetic movement, and writing. Cerebellar inhibition was performed to assess cerebello-thalamo-cortical involvement. DT exhibited higher variability of peak frequency and greater instability of tremor burst intervals over time (higher tremor stability index) than ET or TAWD regardless of tasks. Intermuscular coherence magnitude between the antagonist pairs increased during the writing task in DT, but not ET or TAWD. ET and TAWD exhibited different phase relationships of the temporal fluctuations of voluntary movement and tremor in the kinetic condition. A linear discriminant classifier based on these tremor parameters was able to distinguish the three groups with a classification accuracy of $\mathbf{9 5 . 1 \%}$. Cerebellar inhibition was significantly reduced in DT, but not in TAWD, compared with ET and healthy controls. Our study shows that the two DTS are distinct entities with DT closer to nontremorous dystonia and TAWD closer to ET.

Key words: cerebellar function; dystonic tremor; essential tremor; pathophysiology; transcranial magnetic stimulation

Significance Statement

This study provides novel findings about characteristics and pathophysiology of the two different types of dystonic tremor syndromes compared with essential tremor. Patients with DTS are classified into DT who have dystonia and tremor in the same area, and tremor associated with dystonia (TAWD) who have dystonia and tremor elsewhere. Our results showed that DT exhibits increased tremor variability, instability, and intermuscular coherence, and decreased cerebello-thalamo-cortical inhibition compared with TAWD. Our study shows that DT and TAWD are distinct phenotypes, and that the physiological characteristics of DT are more similar to nontremorous dystonia, and TAWD is closer to ET.

Received May 14, 2020; revised Sep. 14, 2020; accepted Sep. 30, 2020.

Author contributions: P.P. and M.H. designed research; P.P. and H.J.C. performed research; P.P., H.J.C., S.W.L., and M.H. contributed unpublished reagents/analytic tools; P.P., S.W.L., and T.W. analyzed data; P.P. wrote the first draft of the paper; P.P. wrote the paper; S.W.L. and M.H. edited the paper.

This work was supported by the National Institute of Neurological Disorders and Stroke, National Institutes of Health Intramural Program.

The authors declare no competing financial interests.

Correspondence should be addressed to Mark Hallett at hallettm@ninds.nih.gov.

https://doi.org/10.1523/JNEUROSCI.1181-20.2020

Copyright $\odot 2020$ the authors

\section{Introduction}

Dystonic tremor syndromes (DTS) are comprised of tremor and dystonia as the main neurologic signs. Two types of tremor in dystonia are typically classified: dystonic tremor (DT), which is defined as tremor in a body part affected by dystonia, and tremor associated with dystonia (TAWD), defined as tremor in a body part not affected by dystonia (Bhatia et al., 2018). Tremor in dystonia mostly occurs in posture and during voluntary movements resembling essential tremor (Pandey and Sarma, 2016). The clinical characteristics of dystonic tremor are coarse, jerky, irregular, directional, and asymmetrical. Electrophysiological 
studies showed that tremors in both DT and TAWD groups were smaller in magnitude but exhibited higher irregularity in terms of amplitude and frequency compared with essential tremor (ET) (Jedynak et al., 1991; Shaikh et al., 2008; Bove et al., 2018). However, regular oscillations similar to ET have also been demonstrated in various types of DTS (Rudzińska et al., 2013; Shaikh et al., 2015), indicating that there might be overlap in tremor characteristics between DTS and ET.

It is unclear whether tremor in dystonia has its own pathophysiology unrelated to dystonia or shares some mechanisms. Previous neurophysiological studies of tremor in dystonia (both DT and TAWD subtypes) showed that DTS shared similar physiology of dystonia, including loss of inhibition at spinal, brainstem, and cortical level (Munchau et al., 2001; Nistico et al., 2012; Tinazzi et al., 2013; Conte et al., 2018). Functional imaging studies also revealed the involvement of basal ganglia, thalamus, and sensory-motor cortex in DTS, similar to dystonia without tremor (Cerasa et al., 2014; Kirke et al., 2017). Recently, there has been increasing evidence of cerebellar involvement and interactions between cerebellum and basal ganglia in dystonia and DTS. Clinical signs of concomitant cerebellar dysfunction, cerebellar atrophy or lesions, as well as functional abnormalities of cerebellum, and its pathway to the frontal cortex and cerebellar pathology has been shown in dystonic tremor (Ma et al., 2012; Batla et al., 2015; Kirke et al., 2017; Merola et al., 2019). Eye blink classical conditioning, a marker for cerebellar dysfunction, was impaired in patients with cervical dystonia (CD) with tremor (both DT and TAWD), while it was normal in dystonia without tremor (Antelmi et al., 2016). Transient benefit of deep brain stimulation of the ventrointermediate nucleus of thalamus, the nucleus that receives afferents from the cerebellum, also supports the involvement of the cerebello-thalamo-cortical (CTC) pathway and its connections in DTS (Cury et al., 2017). Therefore, the network involving the CTC pathway might play a role in DTS. In addition, the differences in characteristics and pathophysiology of tremor among the subtypes of DTS have never been explored (Defazio et al., 2013). It is still debated whether DT and TAWD are distinct entities or similar syndromes with different types of corresponding dystonia (Defazio et al., 2015).

The primary purpose of the current study was to examine the neurophysiological characteristics of DTS and its subtypes (DT and TAWD) in comparison with ET. Various tremor parameters, including peak frequency (Fp), tremor variability, tremor stability index (TSI) (di Biase et al., 2017), and intermuscular coherence between the antagonist muscle pairs, were investigated and compared between the groups (ET, DT, and TAWD) and with several tasks (postural, kinetic, and writing). The functional influence of the cerebellum or the CTC pathway in DTS can be assessed by cerebellar inhibition (CBI), which quantifies the inhibitory projection from the Purkinje cells to the dentato-thalamo-cortical pathway (Ugawa et al., 1995), being useful for investigating the integrity of the CTC pathway. CBI has been studied in ET with conflicting results, and it was reduced in focal hand dystonia without tremor (Pinto et al., 2003; Molnar et al., 2004; Brighina et al., 2009; Hanajima et al., 2016). Our hypothesis was that DT and TAWD are two distinct subtypes with DT having more CTC dysfunction compared with TAWD and ET.

\section{Materials and Methods}

Participants

Twenty-one patients with a clinical diagnosis of ET (8 females), 22 patients with DTSs (10 females), and 19 age-matched right-handed healthy controls (8 females) without any clinical signs of tremor/ dystonia participated in the study. Patients with DTS were subdivided into 13 patients with DT (writer's cramp with writing tremor) and 9 patients with TAWD (CD with hand tremor). All patients were diagnosed according to the Consensus Statement on Classification of tremor and dystonia (Deuschl et al., 1998; Albanese et al., 2013; Bhatia et al., 2018). Patients were not taking any symptomatic medications for tremor and dystonia for at least 5 half-lives before participation of the study. Those who took medications that may affect the transcranial magnetic stimulation (TMS) results, such as benzodiazepines, primidone, topiramate, selective serotonin-reuptake inhibitors, and antiepileptics, were excluded from the TMS section (Ziemann, 2004). All patients with DTS were studied at least 3 months after botulinum toxin injections. Baseline characteristics and clinical data were collected in all recruited participants and are presented in Table 1. Severity of tremor and its impact on activities of daily living were quantified by The Essential Tremor Rating Assessment Scale (TETRAS) in all patient groups. This scale was used in our study because it focuses mainly on upper extremity action tremor and also assesses head and face tremor that are considered to be the most affected in both ET and DTS (Ondo et al., 2018). Informed consent was obtained before participation. The experiments conformed to the Declaration of Helsinki and were approved by the Institutional Review Board of the National Institutes of Health.

\section{Tremor recordings}

All patients with ET and DTS participated in tremor recordings. Tremor was recorded using two triaxial accelerometers, taped on the dorsum of both hands of the patients with the $z$ axis perpendicular to the dorsal surface. Four pairs of bipolar surface EMG electrodes were placed on the flexor carpi radialis (FCR) and extensor carpi radialis (ECR) muscles bilaterally. These muscles are the antagonist muscle pairs responsible for wrist tremors and are the most severely affected in ET and DTS (both DT and TAWD) (Louis, 2019). Hand tremors were recorded in four conditions: posture $(\mathrm{P})$, posture with 1 pound weighting, simple kinetic movement $(\mathrm{K})$, and writing with the affected hand $(\mathrm{Wr})$. The patients held their hands in an outstretched, horizontal position beyond the edge of the armrest while the pronated forearm was supported on the chair armrest during posture $(\mathrm{P})$, whereas they flexed and extended the hands slowly during simple kinetic movements (K). To evaluate writing tremor, the patients who clinically had tremor while writing were asked to write a spiral or a sentence to elicit tremor with the affected hand. Tremor was recorded for $1 \mathrm{~min}$ and repeated 3 times in each condition.

\section{TMS procedure}

EMG procedure. Participants, including patients and healthy controls, sat in a comfortable chair with their tested arms outstretched and supinated. The tested hand was the dominant side in healthy controls, the more affected hand in patients with ET or TAWD, and both the affected and unaffected side in patients with DT to assess the involvement of the unaffected cortex despite unilateral symptomatic expression in DT. Surface EMG electrodes were placed on the first dorsal interosseus (FDI) muscle in bipolar montages. The EMG signal was amplified using a conventional EMG machine with a bandpass filter between 10 and $2000 \mathrm{~Hz}$

TMS procedure. CBI was obtained from paired-pulse TMS stimulation with conditioning stimulation (CS) delivered to the cerebellar cortex ipsilateral to the recorded hand, followed $5 \mathrm{~ms}$ later by a test stimulus (TS) delivered to the contralateral motor cortex (M1). The TS was applied over the M1 at the FDI hotspot contralateral to the recorded side with a figure-of-eight coil with an external loop diameter of $60 \mathrm{~mm}$, connected to the Bistim2 (Magstim). The TMS procedure was performed similarly to prior work by our group (Panyakaew et al., 2016). Briefly, the location at the FDI hotspot was recorded and maintained throughout the whole experiment using MRI-based neuronavigation. At this location, the input-output curve parameters were obtained to provide the parameters of the resting motor cortex threshold (RMT) and S50. The intensity of the TS was set at S50. S50 is the stimulation intensity that produces a motor evoked potential (MEP) amplitude midway between the EMG baseline and maximum MEP from input-output plots with Boltzmann sigmoidal fitting. 
Table 1. Clinical demographics of ET, DTS, and control subjects ${ }^{\mathrm{a}}$

\begin{tabular}{|c|c|c|c|c|c|c|c|}
\hline & Controls $(N=19)$ & ET $(N=21)$ & DTS $(N=22)$ & $p^{\#}$ & DT $(N=13)$ & $\operatorname{TAWD}(N=9)$ & $p^{\# \#}$ \\
\hline Age (yr) & $55.8 \pm 9.3$ & $56.1 \pm 8.9$ & $59.0 \pm 10.3$ & 0.49 & $59.7 \pm 13.6$ & $55.8 \pm 9.3$ & 0.66 \\
\hline Sex (\% female) & $8(42 \%)$ & $8(38 \%)$ & $10(45 \%)$ & 0.89 & $4(31 \%)$ & $6(67 \%)$ & 0.38 \\
\hline Tremor onset (yr) & - & $21.8 \pm 16.3$ & $36.6 \pm 17.9$ & $0.01^{*}$ & $40.1 \pm 15.9$ & $31.4 \pm 20.2$ & $0.02^{*}(\mathrm{DT}>\mathrm{ET})$ \\
\hline Dystonia onset (yr) & - & - & $44.5 \pm 14.9$ & - & $39.1 \pm 14.4$ & $52.1 \pm 12.8$ & $0.04^{*}$ \\
\hline Dystonia duration (yr) & - & - & $14.5 \pm 14.6$ & - & $20.2 \pm 15.7$ & $6.1 \pm 7.8$ & $0.01^{*}$ \\
\hline Interval between tremor/dystonia onset (yr) & - & - & $-7.9 \pm 15.8$ & - & $1.0 \pm 5.9$ & $-20.7 \pm 17.2$ & $<0.01^{*}$ \\
\hline TETRAS per upper limb & - & $18.3 \pm 4.9$ & $14.7 \pm 6.7$ & 0.06 & $10.2 \pm 4.1$ & $20.8 \pm 4.3$ & $<0.01^{*}(\mathrm{ET}=\mathrm{TAWD}>\mathrm{DT})$ \\
\hline FMS & - & $0 \pm 0$ & $3.1 \pm 2.1$ & $<0.01^{*}$ & $3.7 \pm 2.1$ & $2.1 \pm 1.8$ & $<0.01^{*}(\mathrm{DT}=\mathrm{TAWD}>\mathrm{ET})$ \\
\hline TWSTR & - & $0 \pm 0$ & $5.9 \pm 7.2$ & $<0.01^{*}$ & $2.4 \pm 5.7$ & $10.9 \pm 6.0$ & $<0.01^{*}(\mathrm{TAWD}>\mathrm{DT}=\mathrm{ET})$ \\
\hline
\end{tabular}

${ }^{a}$ DTS patients were subdivided into DT (writer's cramp with writing tremor) and TAWD (CD with hand tremor). ADL, Activity of daily living; per, performance; FMS, Fahn-Marsden Dystonia Rating Scale; TWSTR, Toronto Western Spasmodic Torticollis Rating Scale.

$* p<0.05$.

\# $p$ value from student $t$ test or Mann Whitney $U$ test for continuous variables.

\# $p$ value from one-way ANOVA test or Kruskal-Wallis sum rank test for continuous variables.

For the cerebellar CS, a double-cone coil (110 mm diameter) was positioned over the ipsilateral cerebellum to the recorded hand. The center of the coil was placed $3 \mathrm{~cm}$ lateral and $1 \mathrm{~cm}$ inferior to the inion to stimulate the cerebellar hand representations in lobule VIII of the cerebellum that was confirmed with MRI-based neuronavigation (Daskalakis et al., 2004; Kassavetis et al., 2011). CS intensity was set at the closest $5 \%$ of the stimulator output below the active pyramidal threshold to avoid antidromic corticospinal tract activation that is able to suppress the contralateral motor cortex (Fisher et al., 2009; Ugawa, 2009). The active motor threshold for the pyramidal tract was determined with the double cone coil centered at the inion while subjects made a phasic movement of the FDI (Ugawa et al., 1995; Daskalakis et al., 2004). The coil current direction was downward to induce upward current in the cerebellar cortex. For the best suppression effect, the interstimulus interval between the test and condition stimulus was $5 \mathrm{~ms}$ to explore the function of the CTC pathway in DTS and ET compared with controls (Ugawa et al., 1995; Pinto et al., 2003; Daskalakis et al., 2004); 10 trials were done for TS at M1 alone and 10 trials for the paired-pulse paradigm.

\section{Data analysis}

Tremor analysis: accelerometer and EMG data. The tremor parameters were Fp, half-width power (HWP), FWHM, TSI, intermuscular coherence magnitude (CohM), and intermuscular coherence phase (CohPh). HWP is the area under the curve between two vertical straight lines at the rising and falling edge of the peak at half peak power (FWHM) (Schwingenschuh et al., 2011). HWP is a measure of tremor power (TPtr), whereas FWHM is a measure of the range of tremor frequency or frequency variability. TSI is calculated as the interquartile range of change in frequency, computed from the relationship between instantaneous variation in frequency and instantaneous frequency (di Biase et al., 2017), which was suggested as a neurophysiological parameter that is capable of differentiating ET from PD tremor. The mean of three trials of each parameter was calculated from the dominant hand.

To evaluate the common neural input to the FCR and the ECR muscles (antagonistic pair of hand tremor), the intermuscular coherence values were computed from surface EMG data. The coherence values were estimated from concatenated data (per condition/block) using nonoverlapping segments with a frequency resolution of $1 \mathrm{~Hz}$. Only the coherence values $>95 \%$ confidence limit, determined by the segment length (Halliday et al., 1995), were then $z$-transformed. The integral of $z$ transformed coherence between 8 and $55 \mathrm{~Hz}$ was estimated, which includes the three frequency bands of interest: $\alpha(8-12 \mathrm{~Hz}), \beta(13-$ $35 \mathrm{~Hz})$, and $\gamma(36-55 \mathrm{~Hz})$, each of which represents common neural input to the muscles via different pathways (spinal, corticospinal, and subcortical, respectively) (Farmer et al., 1993; Norton et al., 2003; Nishimura et al., 2009). For each condition/group, the CohM and $\mathrm{CohPh}$ values were computed.
Time frequency characteristics of the simple kinetic movements were analyzed to examine the effects of voluntary movement on tremor. First, the time-frequency analysis was performed on the accelerometer data by applying a moving window of $1 \mathrm{~s}$ duration with $0.5 \mathrm{~s}$ overlapping to compute the power spectrum over time. Then, at each time point, the TPtr was computed as the peak power (in decibels) between 4 and $10 \mathrm{~Hz}$, and the movement power (TPmov) as the peak power between 0 and $4 \mathrm{~Hz}$. TPtr and TPmov quantify the degree of fluctuation in the tremor and that of the voluntary movement over time, respectively. Temporal synchronization of fluctuation in TPtr and that in TPmov was then assessed by computing the coherence between the TPtr and the TPmov at the frequency of the power fluctuation for each subject (typically observed between 0.1 and $0.3 \mathrm{~Hz}$ ). The confidence limit of the coherence values between the TPmov and TPtr were computed from the segment length used to estimate the coherence values (Halliday et al., 1995), and only the coherence values (at the power fluctuation frequency) greater than the confidence limit was considered in the analysis. The magnitude of the coherence values indicates the degree of synchronization of the temporal fluctuation of the tremor intensity/power and that of the voluntary movement, while the coherence phase quantifies the temporal delay between the TPmov and TPtr fluctuations.

TMS data analysis. MEP amplitudes of the recorded FDI muscle were measured peak-to-peak in each trial in millivolts. The mean MEPs of 10 trials of test alone and paired-pulse stimulation were calculated. $\mathrm{CBI}$ is a ratio of mean conditioned MEP and unconditioned MEP. CBI changes were compared between groups and subgroups.

\section{Statistical analysis}

Baseline characteristics, tremor parameters, and TMS parameters were summarized using either means and SDs, or frequencies and percentages as appropriate. The Shapiro-Wilk test was performed to evaluate the normality of demographics and parameters in each group. Differences in demographics and TMS parameters, including CBI ratio among ET, DTS, and controls, were evaluated using the one-way ANOVA with two-sample $t$ tests or Kruskal-Wallis rank sum test with Mann-Whitney test for post hoc pairwise tests for continuous variables. Bonferroni's adjustment was used for multiple comparisons between groups. To compare between subgroups (DT, TAWD, ET, and controls), one-way ANOVA with two-sample $t$ tests or Kruskal-Wallis rank sum test with Mann-Whitney test for post hoc pairwise tests was also performed. Correlation between CBI ratio and tremor severity scale was evaluated with Pearson's correlation test. The mixed model repeated ANOVA was used to test the differences in tremor characteristics in different conditions among patient groups. Log transformation was applied for HWP and FWMH before performing the mixed model repeated ANOVA because these two parameters were not normally distributed. The experiment in the tremor section had three within-subject factors (conditions; posture, kinetic, and writing) and three between-subject factors (groups; 

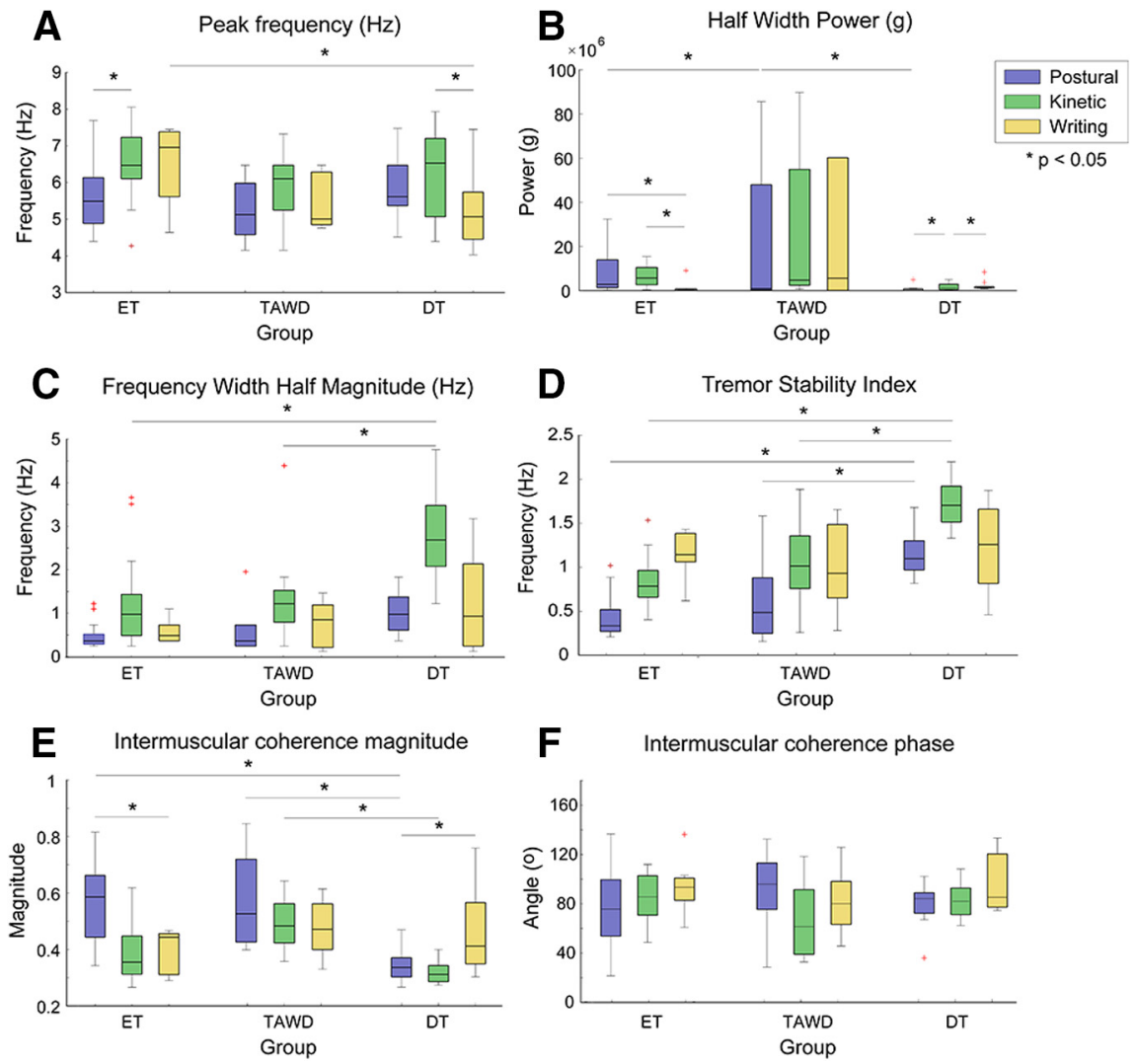

Figure 1. Tremor characteristics. Box-and-whisker plots represent median with interquartile range of postural, kinetic, and writing conditions across ET, DT, and TAWD. A, Fp (Hz). B, HWP (g). C, FWHM (Hz). D, TSI. E, Intermuscular CohM. F, CohPh.

ET, DT, and TAWD). Condition, group, and the interaction between condition and group were fixed effects. Simple $t$ test was used for post hoc pairwise tests with Bonferroni's adjustment for multiple comparisons. Statistical analysis was performed using SPSS version 23.0 software (IBM). Cohen's $d$ was computed to report effect sizes when the pairwise comparisons showed significant differences.

Classification of patient groups using tremor characteristics

The tremor parameters that were found to be significantly different between groups were used to build different classifiers with linear discriminant analysis (LDA) to distinguish among three patient groups. LDA projects the features to a space that minimizes within-group variability and maximizes between-group variability by finding eigenvalues of the between-class variance matrix (normalized by the within-class variance).

Two types of validation for the LDA classifier were performed. For the interpolation, data points from all subjects were used to build the model; then the performance of the model was validated against the same dataset. For the extrapolation, the classifier was built, excluding data from one subject, which was then implemented to identify the group of the excluded subject.

Additionally, we performed leave-one-out cross validation of the tremor parameters to evaluate their contribution to the classification accuracy. Namely, for each tremor parameter identified with a significant between-group difference, an LDA classifier was built without including the parameter and its classification accuracy was estimated. The lower the classification accuracy, the more significant is the contribution of the parameter to the between-group separation.

\section{Results}

\section{Patient characteristics}

In our study, patients with DTS were significantly older at onset of tremor $(36.6 \pm 17.9$ vs $21.8 \pm 16.3, p=0.01$; effect size $d=$
0.87 ) and had less tremor severity (TETRAS performance scale; $14.9 \pm 7.9$ vs $19.8 \pm 5.9, p=0.03$; effect size $d=0.73$ ) than patients with ET (Table 1). When DTS was subdivided into DT and TAWD, tremor onset and tremor severity were different between DT and ET but not between TAWD and ET. Tremor started at or after dystonia onset in DT, while tremor started before dystonia onset in TAWD (interval between tremor and dystonia onset; $1.0 \pm 5.9$ vs $-20.7 \pm 17.2, p<0.001$; effect size $d=1.84$ ).

\section{Tremor characteristics: accelerometry and EMG data}

Data from all patients were used in the tremor analysis, except 1 subject in the DT group, who was excluded because of intermittent tremor during tremor recording. Writing tremor was recorded only in 7 ET, 12 DT, and 6 TAWD patients who clinically had tremor during writing. The tremor characteristics across three groups in each condition (posture, kinetic, writing) are shown in Figure 1. Fp was found significantly different across conditions $\left(F_{(2,62.8)}=6.99, p=0.002\right)$ (Fig. 1A). There was no significant effect of group $\left(F_{(2,40.2)}=2.28, p=0.11\right)$, but the interaction between condition and group was found significant $\left(F_{(4,62.7)}=3.14, p=0.02\right)$. Post hoc pairwise comparisons showed that, while the Fp of kinetic tremor was the highest for all groups, significant differences were found between kinetic and postural tremor in ET $(p<0.001$; effect size $d=1.00)$ and between kinetic and writing tremor in DT ( $p=0.006$; effect size $d=0.94)$. When the Fp of writing tremor was compared among patient groups, the Fp value was significant higher in ET than DT $(p=0.02$; effect size $d=1.15$ ).

Additionally, Fp of posture with 1 pound weighting condition was compared with that of the posture $(\mathrm{P})$ condition for each 
group to determine the nature of oscillator whether its origin is central or peripheral (Deuschl et al., 2001). The difference of Fp between $\mathrm{Wt}$ and $\mathrm{P}$ was $<1 \mathrm{~Hz}$ in all groups (ET; Fp $\mathrm{P}=$ $5.65 \pm 0.89$ vs Fp Wt $=5.59 \pm 1.0$, TAWD; Fp P $=5.25 \pm 0.84$ vs $\mathrm{Fp} \mathrm{Wt}=5.13 \pm 0.97, \mathrm{DT}$; Fp P $=5.86 \pm 0.96$ vs Fp Wt $=4.89 \pm$ $0.48)$, indicating central tremor.

When tremor magnitude (HWP) was compared among conditions and groups, there were significant effects of condition $\left(F_{(2,60.7)}=15.9, p<0.001\right)$ and the interaction between condition and group $\left(F_{(4,60.7)}=12.79, p<0.001\right)$, but the effect of group $\left(F_{(2,39.1)}=2.03, p=0.15\right)$ was not found significant (Fig. 1B). Post hoc pairwise comparison showed that HWP of postural tremor was significantly lower in DT compared with ET ( $p=0.006$; effect size $d=0.88)$ and TAWD $(p=0.03$; effect size $d=0.81)$. Nevertheless, HWP of writing tremor was not different among groups. For ET, HWP of postural and kinetic tremor was higher than writing tremor $(p=0.006$; effect size $d=0.72, p=0.03$; effect size $d=0.81$, respectively); whereas for DT, HWP of postural tremor was lower than kinetic and writing tremor $(p=0.006$; effect size $d=0.71, p=0.006$; effect size $d=0.76$, respectively).

FWHM was analyzed to determine tremor variability in each condition and group (Fig. 1C). The main effects for group $\left(F_{(2,40.2)}=9.11, p=0.001\right)$ and condition $\left(F_{(2,66.6)}=16.2\right.$, $p<0.001)$ were significantly different, while the interaction between condition and group was not significantly different $\left(F_{(4,66.5)}=0.74, p=0.57\right)$. Post hoc pairwise comparison revealed that FWHM was significantly greater in DT than ET $(p<0.001$; effect size $d=0.93$ ) and TAWD ( $p=0.01$; effect size $d=0.67$ ). The FWHM of kinetic tremor was the highest compared with postural $(p<0.001$; effect size $d=0.82)$ and writing tremor ( $p=0.01$; effect size $d=0.48)$ in all groups.

The TSI, a measure of stability of tremor frequency over time, was analyzed and compared among patient groups (Fig. 1D). Interestingly, both main effects for group $\left(F_{(2,38.7)}=20.26\right.$, $p<0.001)$, condition $\left(F_{(2,64.3)}=21.48, p<0.001\right)$, and the interaction between condition and group $\left(F_{(4,64.2)}=4.56, p=0.003\right)$ were significantly different. Post hoc pairwise comparison demonstrated that TSI of postural and kinetic tremor was significantly greater in DT compared with ET $(p<0.001$; effect size $d=2.30)$ and TAWD $(p<0.001$; effect size $d=1.34)$. The pattern of TSI of three different conditions was not similar to that of FWHM. We observed that the highest TSI was during writing in ET, whereas TSI during writing was not higher than kinetic in both TAWD and DT. However, TSI of writing tremor was not significantly different among three groups.

To evaluate the intermuscular coherence between of the FCR and the ECR muscle as the antagonist pair of hand tremor, the CohM and CohPh were extracted from surface EMG data in each condition (Fig. 1E,F). For CohM, there were significant effects for group $\left(F_{(2,36.5)}=7.53, p=0.002\right)$, condition $\left(F_{(2,61.6)}=\right.$ $8.57, p=0.001)$, and the interaction between condition and group $\left(F_{(4,61.5)}=7.05, p<0.001\right)$. Post hoc pairwise comparison showed that CohM of postural tremor was significantly different between DT and ET $(p<0.001$; effect size $d=2.09)$ and between DT and TAWD ( $p=0.006$; effect size $d=2.23$ ). CohM of postural tremor was significantly higher than kinetic $(p<0.001$; effect size $d=1.55)$ and writing $(p<0.001$; effect size $d=1.41)$ in ET while CohM of writing tremor was significantly higher than postural $(p=0.04$; effect size $d=1.12)$ and kinetic tremor $(p=0.006$; effect size $d=1.49$ ) in DT. For TAWD, a similar pattern with ET was observed but did not reach statistical significance ( $\mathrm{P}$ vs $\mathrm{K}$; $p=0.10, \mathrm{P}$ vs $\mathrm{W} ; p=0.05)$. In contrast to CohM, the main effects for group $\left(F_{(2,38.3)}=0.27, p=0.77\right)$ and condition $\left(F_{(2,62.8)}=2.05\right.$, $p=0.14)$ and interaction between condition and group were not significantly different $\left(F_{(4,62.7)}=1.62, p=0.18\right)$ in CohPh. We did not observe any consistent patterns of synchronous or cocontraction of the antagonist pair in any group of patients.

\section{Effect of voluntary movement on tremor}

Temporal fluctuation in $\operatorname{TPtr}\left(\mathrm{F}_{\mathrm{tr}}\right)$ was evaluated in each condition and compared among groups. There were significant effects for condition $\left(F_{(2,61.4)}=4.61, p=0.01\right)$ and the interaction between condition and group $\left(F_{(4,61.4)}=2.96 p=0.03\right)$, but there was no significant effect of group $\left(F_{(2,39.0)}=0.7, p=0.50\right)$. Post hoc pairwise comparison demonstrated a significant difference in $\mathrm{F}_{\text {tr }}$ between postural and kinetic tremor in ET $(p=0.001$; effect size $d=0.83$ ).

We further explored the effect of voluntary movement on tremor during simple kinetic movement in patients with ET, TAWD, and DT because only simple cyclic movement (kinetic condition) affected temporal fluctuation of tremor but not writing. When a voluntary movement was made, it affected tremor of the patient groups differently (Fig. $2 A-C$ ). The interaction between voluntary movements and tremors in the kinetic condition was significantly different among groups (one-way ANOVA; $\left.F_{(2,3.92)}=7.53, p=0.03\right) . F_{\text {tr }}$ was more synchronized with that of TPmov in TAWD, indicated by significant smaller phase delay in coherence between TPtr and TPmov (phase delay $=57.1 \pm 15.6^{\circ}$ ), compared with the phase delay in ET patients (phase delay = $109.6 \pm 9.6^{\circ}$; post hoc pairwise tests with Bonferroni's adjustment, $p=0.025$; effect size $d=1.21$ ) (Fig. 3 ).

\section{Cluster analysis of tremor characteristics to distinguish among groups}

The following tremor characteristics from the dataset were found to be significantly different across groups and, thus, were used to build different classifiers: (1) Fp in power spectrum, (2) FWHM, (3) TSI, (4) phase delay between temporal fluctuation of TPmov $(0-4 \mathrm{~Hz})$ and $\operatorname{TPtr}(4-10 \mathrm{~Hz})$, and (5) intermuscular CohM between the ECR and FCR muscle.

The LDA classifier based on these features was able to distinguish among ET, DT, and TAWD with a high accuracy. The accuracy of LDA classification was $95.1 \%$ for the interpolation and 85.4\% for the extrapolation (Fig. 4).

Additionally, results from the leave-one-out cross validation of the tremor parameters showed that the CohM contributed the most to the between-group separation (41.5\% classification accuracy by the LDA classifier built without CohM), and the Fp the least (90.2\% accuracy by the classifier without Fp).

\section{CBI reduction in DTS compared with controls}

A total of 18 ET, 20 DTS, and 19 controls completed the TMS experiment. Three ET and 2 TAWD patients were excluded because of inability to tolerate cerebellar stimulation (1 patient) and taking medications that might affect TMS results (4 patients). The TMS parameters and CBI results are shown in Table 2. The mean RMT at the motor cortex and TS were not significantly different among patients and controls $(p=0.37$, $p=0.77$, respectively), while the pyramidal threshold and CS were significantly lower in DTS than controls (DTS vs controls; $p=0.03$; effect size $d=0.85, p=0.02$; effect size $d=0.97$, respectively). There is no clear explanation for the lower pyramidal threshold in DTS compared with controls, and an earlier study did not find any difference (Brighina et al., 2009). However, the MEP sizes with TS alone that might influence the CBI result were similar across groups $(p=0.13)$. 
A
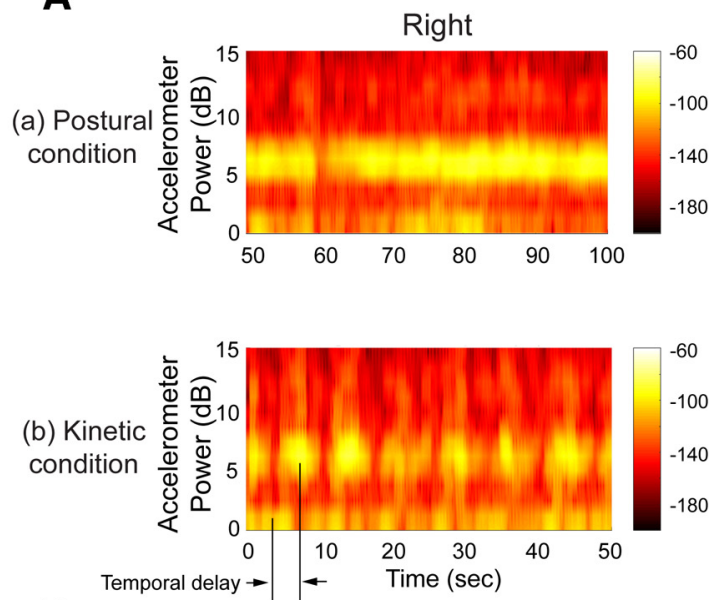

B Movement power Tremor power
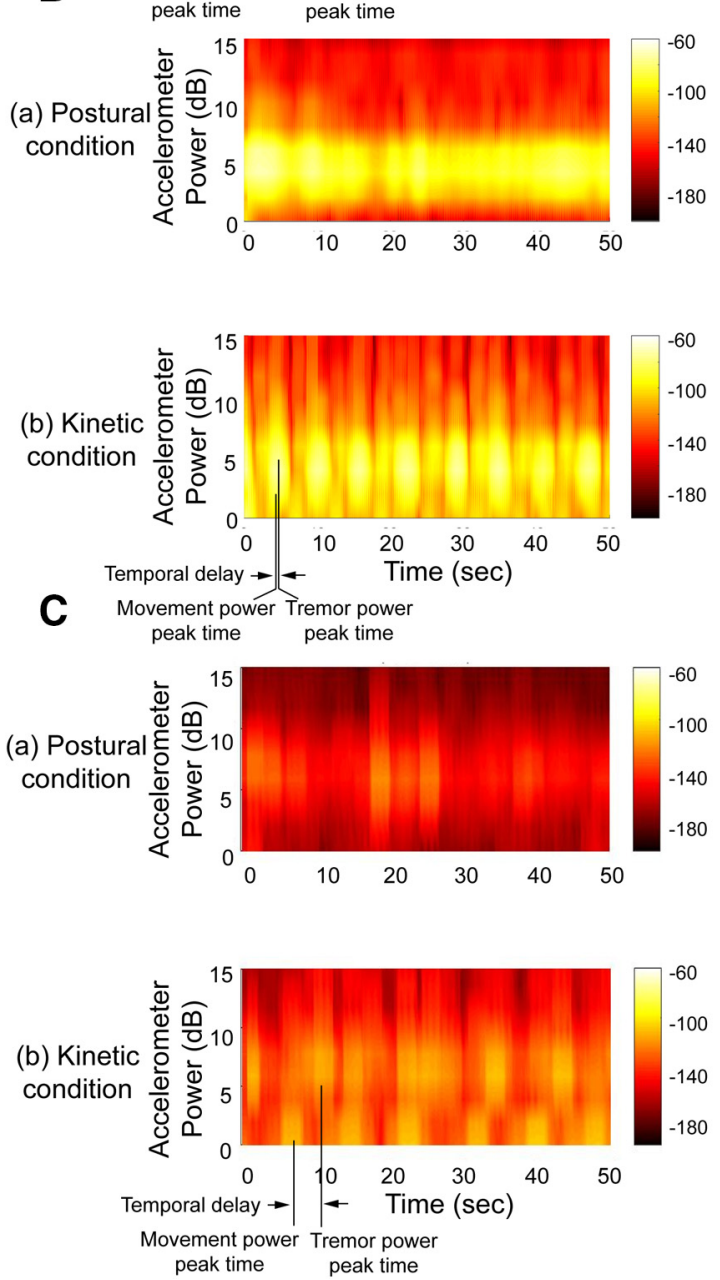
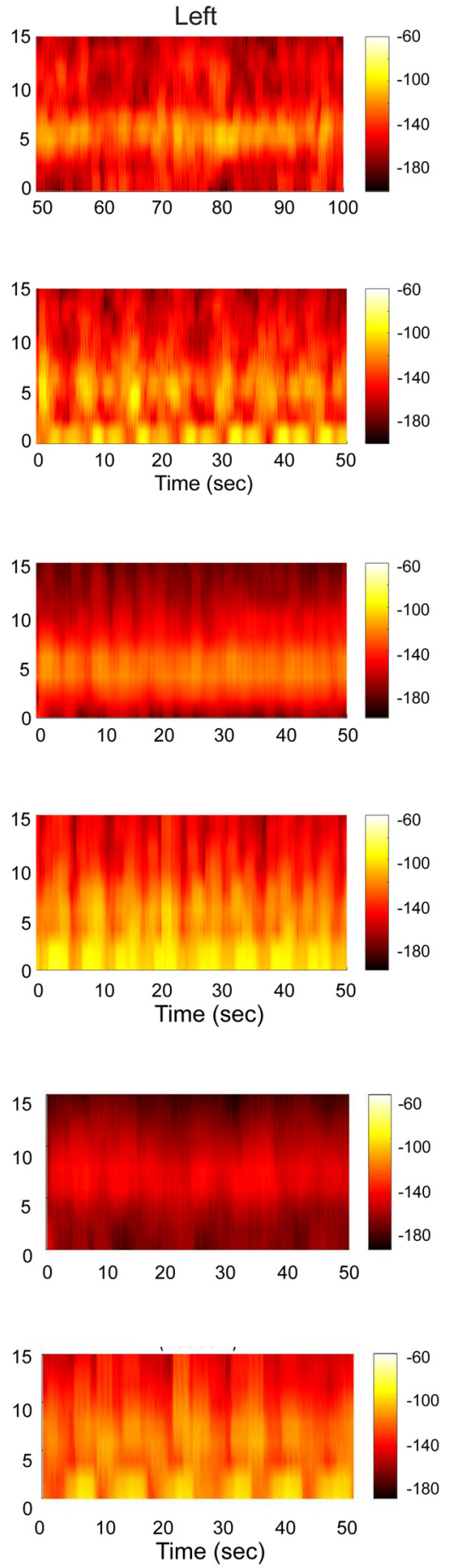

Figure 2. Representative cases of the time-frequency analysis for postural and kinetic tremor in $(\boldsymbol{A}) \mathrm{ET},(\boldsymbol{B}) \mathrm{TAWD}$, and $(\boldsymbol{C})$ DT. There was a large degree of temporal (phase) delay between the fluctuation of the tremor $(4-10 \mathrm{~Hz})$ and movement $(0-4 \mathrm{~Hz})$ peak powers in the kinetic condition in ET and DT (out-of-phase), whereas there was less phase delay (more synchronous) between tremor and movement in TAWD (in-phase).

CBI was calculated as a ratio of mean conditioned MEPs and unconditioned MEPs. There was a significant difference in CBI ratio among groups $\left(F_{(2,54)}=4.38, p=0.02\right)$ as shown in Figure $5 A$. Post hoc pairwise comparison showed that CBI was significantly reduced (less inhibition) in patients with DTS compared with controls ( $p=0.03$; effect size $d=0.73$ ) but not ET compared with controls $(p=0.94)$.

\section{CBI reduction in DT compared with controls and ET but not TAWD}

Twenty patients with DTS were subdivided into 13 DT and 7 TAWD and compared with ET and control. There were no significant differences in the mean RMT at the motor cortex, TS, pyramidal threshold, CS, and the MEP sizes with TS alone among patient groups and controls. Subgroup analysis revealed a 


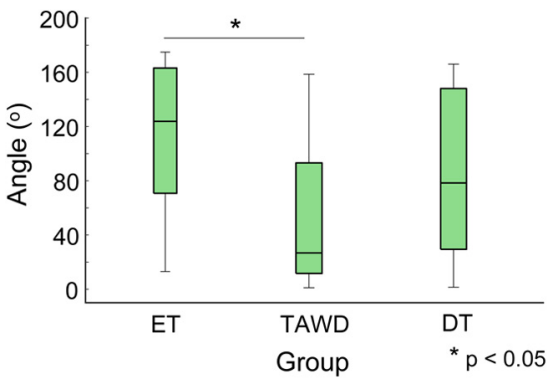

Figure 3. The phase delay (box-and-whisker plots; median with interquartile range) in coherence between TPtr and TPmov in ET, TAWD, and DT.

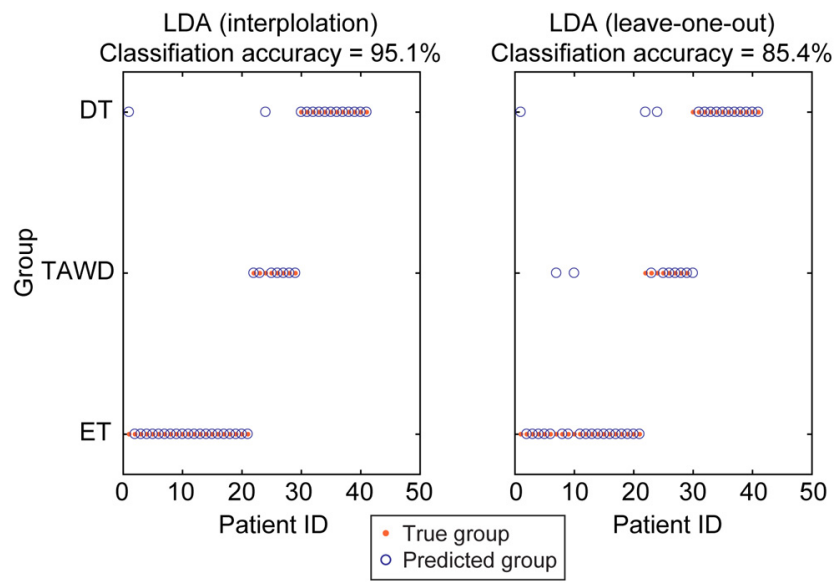

Figure 4. Cluster analysis of tremor characteristics to distinguish among ET, DT, and TAWD. The accuracy of LDA classification was $>95 \%$ (interpolation method) and $>85 \%$ (leave-one-out method).

significant difference in CBI across groups $\left(F_{(3,53)}=4.05\right.$, $p=0.01$ ) as shown in Figure $5 B$. Post hoc pairwise comparison demonstrated a significant CBI reduction in DT, but not TAWD, compared with controls (DT vs controls; $p=0.01$; effect size $d=1.20$ ) and ET (DT vs ET; $p=0.04$; effect size $d=1.16$ ). When CBI on the unaffected side in patients with DT was added to the post hoc pairwise comparison, it was not significantly different from the affected side $(p=0.84)$.

\section{Correlation between $\mathrm{CBI}$ and tremor severity scale}

Exploratory correlation analysis was performed to evaluate the contribution of the cerebellum in tremor genesis. There was a negative correlation between CBI and TETRAS performance scale only in the patients with ET $(r=-0.57, p=0.01)$ but not DTS $(r=-0.06, p=0.78$ ) (Fig. $5 C$ ). CBI was not significant correlated with TETRAS scale either in DT $(r=0.004, p=0.99)$ or TAWD $(r=0.34, p=0.45)$ subgroup.

\section{Discussion}

Our study examined the neurophysiological characteristics of dystonic tremor and its subtypes. Tremor in DT revealed higher variability and increased instability than ET and TAWD regardless of conditions. TAWD shared more tremor characteristics with ET compared with DT. However, ET and TAWD had a different degree of interaction between voluntary movements and tremor oscillators during the simple kinetic condition. DT had less inhibition in the CTC pathway than ET or TAWD. A correlation was found between $\mathrm{CBI}$ and tremor severity scale only in
ET. These results fit our hypothesis that DTS is comprised of two distinct subtypes with different physiologies, with DT having more reduced CTC function.

The relationship between dystonia and tremor onset was different between DT and TAWD. We found that tremor started at or after dystonia onset in DT, while tremor started before dystonia onset in TAWD. This result differs from a previous DTS cohort in which tremor started at or after onset of dystonia in both DT and TAWD (Defazio et al., 2013). However, Schiebler et al. (2011) showed similar findings to ours that one subgroup of patients with $\mathrm{CD}$ and hand tremor had tremor starting before onset of CD. This group of patients had higher severity of tremor compared with typical DT, like ET. This supports the idea that baseline characteristics of TAWD are more like ET or an ET syndrome tainted with dystonia. However, a larger cohort should be done to prove this concept.

Tremor in DT had higher FWHM and TSI values compared with ET and TAWD, indicating increased variability in tremor frequency and instability of tremor in all conditions. The interpretation of FWHM is different from TSI. TSI measures the range of cycle to cycle changes over time, which can precisely capture the short-term stability or temporal aspect of tremor. Conversely, FWHM detects the range of different frequencies within the signal, as it is derived from examining the power spectrum of the entire signal (not from cycle-to-cycle variations). The TSI can capture the time-varying behavior of a single oscillator, whereas FWHM might better characterize the shifting between multiple oscillators as has been previously proposed (Shaikh et al., 2008; Brittain et al., 2015; di Biase et al., 2017). In DT, there could be a number of oscillators with irregularity in both amplitude and frequency (Deuschl et al., 2001), or a superimposed dystonic contraction might disrupt tremor rhythmicity, leading to increases both in FWHM and TSI (Bove et al., 2018). An alternative hypothesis for increased tremor instability in DT might be that the results depend on tremor amplitude since there were differences in tremor amplitude among groups. Stronger tremor possibly comes from greater entrainment of central oscillators, leading to more stability. Also, a significant negative correlation between tremor amplitude and TSI $(r=-0.46, p<0.01)$ was found in our study. An analysis adding tremor amplitude as a covariate was performed to address this hypothesis. The results were similar (data not shown), suggesting that higher tremor instability in DT compared with TAWD and ET is because of its underlying pathophysiology. Our data showed that TSI was dependent on conditions, contrary to a previous report (di Biase et al., 2017). Tremor possibly becomes more unstable when motor commands interact with oscillators while performing a complex task. TSI of writing tremor was the highest in ET compared with kinetic and postural condition, which points to more involvement of cerebellum as a part of feedforward loop while performing complex motor task in ET (Alahmadi et al., 2016). This hypothesis was supported by the delayed onset of the second agonist during triphasic pattern of ballistic movement in ET resembling cerebellar disorders (Munchau et al., 2001). Moreover, tremor in ET is more unstable under conditions that require increased visual feedback such as writing (Archer et al., 2018).

Intermuscular CohM of writing was increased in DT compared with other conditions. Indeed, this finding supports dystonic cocontraction and relates to the loss of reciprocal inhibition that has been widely described in dystonia (Hallett, 2011; Gallea et al., 2018; Quartarone and Ruge, 2018), suggesting that there is some degree of pathophysiological overlap between DT and dystonia without tremor. TAWD was shown to share some 
Table 2. TMS Parameters and CBI results of ET patients, DTS patients, and control subjects (C) ${ }^{\mathrm{a}}$

\begin{tabular}{lccclccc}
\hline & Controls $(\boldsymbol{N}=\mathbf{1 9})$ & $\mathbf{E T}(\boldsymbol{N}=\mathbf{1 8})$ & $\mathbf{D T S}(\boldsymbol{N}=\mathbf{2 0})$ & $\boldsymbol{p}^{\#}$ & DT $(\boldsymbol{N}=\mathbf{1 3})$ & TAWD $(\boldsymbol{N}=\mathbf{7})$ & $\boldsymbol{p}^{\#}$ \\
\hline RMT & $47.3 \pm 9.9$ & $46.5 \pm 9.8$ & $44.3 \pm 12.1$ & 0.37 & $44.1 \pm 12.7$ & $44.7 \pm 11.9$ & 0.58 \\
TS intensity & $59.2 \pm 11.8$ & $58.3 \pm 12.0$ & $56.4 \pm 12.1$ & 0.77 & $56.6 \pm 12.8$ & $56.1 \pm 11.8$ & 0.91 \\
Pyramidal threshold & $64.1 \pm 10.9$ & $58.8 \pm 10.5$ & $54.5 \pm 11.7$ & $0.04^{*}(C>$ DTS $)$ & $55 \pm 11.2$ & $56.1 \pm 11.8$ & 0.09 \\
CS intensity & $61.3 \pm 12.3$ & $53.8 \pm 10.5$ & $49.7 \pm 11.7$ & $0.02^{*}(C>$ DTS $)$ & $50.4 \pm 11.3$ & $48.6 \pm 13.5$ & 0.05 \\
MEP sizes of the FDI & $2.0 \pm 1.2$ & $3.3 \pm 2.5$ & $3.2 \pm 2.2$ & 0.13 & $3.5 \pm 2.6$ & $2.5 \pm 1.1$ & 0.22 \\
CBI of the FDI & $0.75 \pm 0.17$ & $0.77 \pm 0.15$ & $0.86 \pm 0.13$ & $0.02^{*}$ (DTS > C) & $0.91 \pm 0.08$ & $0.77 \pm 0.15$ & $0.01^{*}$ (DT > ET) (DT > C)
\end{tabular}

${ }^{a}$ DTS patients were subdivided into DT (writer's cramp with writing tremor) and TAWD (CD with hand tremor). MSO, Maximum stimulus output.

$* p<0.05$.

\# $p$ value from one-way ANOVA test or Kruskal-Wallis sum rank test for continuous variables.

A

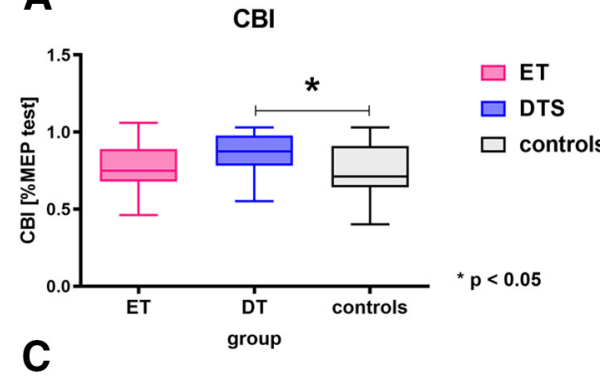

CBI and tremor severity score

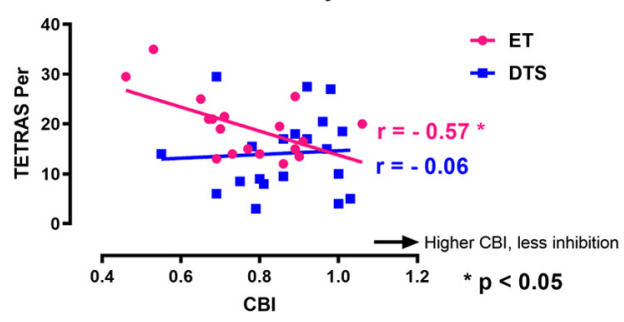

B

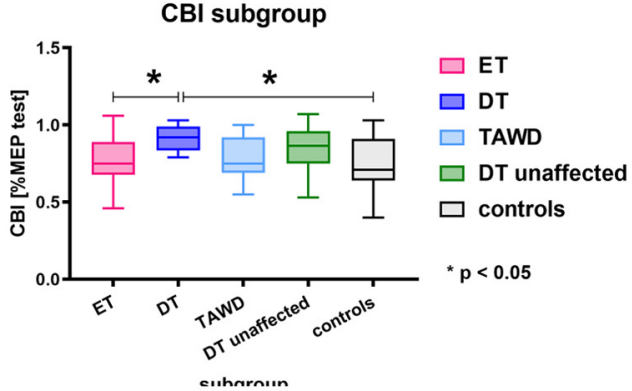

$\mathrm{CBI}$ and tremor severity score

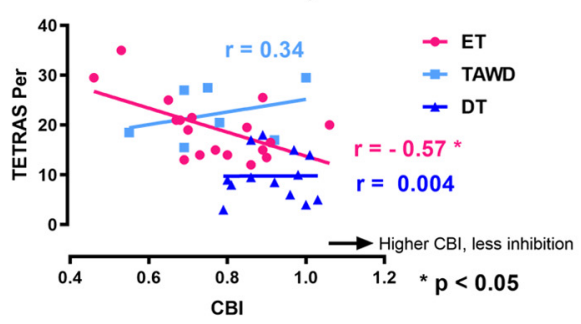

Figure 5. Summary of $C B I$ (box-and-whisker plots; median with interquartile range) in ET, DTS, and controls ( $\boldsymbol{A}$ ) and in subgroups of DTS, DT and TAWD, and the unaffected side of DT (B). Correlation analysis between (BI and TETRAS performance scale (C) in ET, DTS, and subgroups of DTS (D).

tremor characteristics, including tremor variability, instability, and CohM with ET rather than DT, perhaps the characteristics of TAWD could be considered as ET tainted with a bit dystonia. However, the effect of voluntary movements on tremor was different between ET and TAWD. $F_{\text {tr }}$ was close to synchronized with that of TPmov in TAWD, whereas it was close to alternating or out of phase in ET. For TAWD, a similar pathway might be used for movement and tremor production because of loss of inhibition and abnormal sensorimotor integration (Tinazzi et al., 2013). In contrast, for ET, competing pathways or oscillators might be used instead. This could imply that TAWD still has some overlap with the physiology of dystonia. Increased FWHM, TSI, and CohM in DT compared with TAWD and ET could be because of a number of final output oscillators with irregular properties, loss of reciprocal inhibition, and dystonic cocontraction. The differences in these parameters strongly suggest that the phenotype of DT and TAWD differs significantly. In summary, the combination of Fp, FWHM, TSI, CohM, and phase delay between temporal fluctuation of TPmov and TPtr is capable of separating the different tremor groups, pointing to a possible role of these tremor parameters in differential diagnosis. Intermuscular coherence contributes the most to distinguish between tremor types, indicating that CohM might have a promising diagnostic value.

CBI was significantly reduced in DT, but not in TAWD, compared with ET and controls, indicating that DT had less inhibition in the CTC pathway. Impaired CBI has been described in focal hand dystonia without tremor (Brighina et al., 2009), which suggests an involvement of the cerebellum in pathophysiology of dystonia (Shakkottai et al., 2017). The possible explanation for reduced CBI in DT might be because of dystonia, not the "tremor part." This possibility suggests that cerebellar dysfunction previously described in DT (Batla et al., 2015; Antelmi et al., 2016; Kirke et al., 2017; Merola et al., 2019) might be the part of cerebellar involvement in dystonia. As virtually all tremors involve the cortico-cerebello-thalamo-cortical loop, a difference in the CTC pathway in DT may well influence its production. A recent functional neuroimaging study showed that there was widespread cerebello-basal ganglia-cortical activity associated with tremor in DT compared with ET (DeSimone et al., 2019). Reduced CBI in both affected and unaffected sides in DT supports this widespread network hypothesis.

TAWD has normal CBI, similarly to ET and controls. Both normal and reduced CBI have been described in ET (Pinto et al., 2003; Hanajima et al., 2016). The difference might be the location of cerebellar stimulation and different subgroups of ET in two studies. However, in our method, the MEP sizes of TS and CBI results are more consistent with Pinto et al. (2003), pointing to disturbances of the olivocerebellar pathway in ET (Iwata and Ugawa, 2005; Grimaldi et al., 2014). The negative correlation between $\mathrm{CBI}$ and the tremor severity scale may indicate that there is more influence of the cerebellum when tremor is worse 
in ET but not TAWD. However, generalization of this correlation might be limited because of a small number of patients with various tremor severity in each group. The different CBI results between DT and TAWD might not be simply because of different types of focal dystonia. CBI was reduced in focal hand dystonia without tremor, whereas it was either reduced or normal in CD without hand tremor (Brighina et al., 2009; Koch et al., 2014; Porcacchia et al., 2019). Recent evidence suggests that the pathophysiological role of the cerebellum is not identical in all types of dystonia (Bologna et al., 2016). Thus, reduced CBI in DT opposed to that in TAWD could be because of different integrity of the CTC pathway in a dystonia network or its different function in the tremor circuit between DT and TAWD. The corticocerebello-thalamo-cortical loop seems to be involved in every type of tremor (Nieuwhof et al., 2018). The network for tremor genesis might be similar in DT, TAWD, and ET, but the differences would be because of the different integrity of the CTC segment as a part of the circuits among groups. The CTC functional connection might be weaker in DT contributing to its instability. There might also be some basal ganglia influence on the network and that might be more in dystonic tremor than ET. Basal ganglia can be linked to the cortico-cerebello-thalamo-cortical loop in a similar way to that of PD tremor (Helmich et al., 2012; Dirkx et al., 2016). Reduced CBI in DT similar to PD tremor also supports the idea that the basal ganglia could interact with the CTC pathway contributing to tremor in DT. Tremor can differ in their phenotype or their pathophysiology. The findings in our study highlight the distinct phenotypes of DT and TAWD in detail with good separation of tremor types. Multiple different underlying pathophysiologies between DT and TAWD, including the different properties of oscillators, the different CTC involvement, or even a dystonic cocontraction of the antagonist pairs at the body part where tremor is manifested, might contribute to the different phenotypes of the tremors. Thus, our findings add some new information about the fundamental pathophysiology and lead to therapeutic implications.

A strength of our study is the careful selection of patients to be homogeneous in each group. In contrast to the prior studies that combined patients with DTS, we intentionally selected and subdivided DTS into DT and TAWD to explore the differences of physiology between subgroup. The number of patients in each group is small; however, we can still demonstrate differences in physiologic characteristics among groups. A future study with larger sample size should be done to replicate the result. Our study had a limitation of using only $5 \mathrm{~ms}$ interstimulus intervals for CBI experiment. Possible different effects of CBI at other interstimulus intervals among patient groups and controls could not be detected.

In conclusion, our study provides novel evidence that DT and TAWD are distinct entities in the spectrum of DTS with different tremor characteristics. DT might have a number of oscillators with irregular properties with increased instability and/or the superimposition of dystonic contraction with increased coherence because of loss of reciprocal inhibition, indicating that DT shares some dystonia physiology. DT also has less inhibition in the CTC pathway than ET and TAWD reflecting the dystonia, not necessarily the tremor. The involvement of the CTC pathway may well be an important difference between DT and TAWD/ ET. This suggests that CTC integrity as a part of the tremor circuit would be weaker in DT allowing the basal ganglia to have a more important influence on these networks while the CTC functional connection is stronger in TAWD similar to ET. The characteristics of tremor in TAWD are in between ET and DT and lead to different therapeutic implications in each subtype of DTS. Treatment of dystonia could be applied for DT while antitremor medications that are commonly used in ET would be suitable for TAWD.

\section{References}

Albanese A, Bhatia K, Bressman SB, Delong MR, Fahn S, Fung VS, Hallett M, Jankovic J, Jinnah HA, Klein C, Lang AE, Mink JW, Teller JK (2013) Phenomenology and classification of dystonia: a consensus update. Mov Disord 28:863-873.

Alahmadi AA, Samson RS, Gasston D, Pardini M, Friston KJ, D’Angelo E, Toosy AT, Wheeler-Kingshott CA (2016) Complex motor task associated with non-linear BOLD responses in cerebro-cortical areas and cerebellum. Brain Struct Funct 221:2443-2458.

Antelmi E, Di Stasio F, Rocchi L, Erro R, Liguori R, Ganos C, Brugger F, Teo J, Berardelli A, Rothwell J, Bhatia KP (2016) Impaired eye blink classical conditioning distinguishes dystonic patients with and without tremor. Parkinsonism Relat Disord 31:23-27.

Archer DB, Coombes SA, Chu WT, Chung JW, Burciu RG, Okun MS, Wagle Shukla A, Vaillancourt DE (2018) A widespread visually-sensitive functional network relates to symptoms in essential tremor. Brain 141:472485.

Batla A, Sánchez MC, Erro R, Ganos C, Stamelou M, Balint B, Brugger F, Antelmi E, Bhatia KP (2015) The role of cerebellum in patients with late onset cervical/segmental dystonia? Evidence from the clinic. Parkinsonism Relat Disord 21:1317-1322.

Bhatia KP, Bain P, Bajaj N, Elble RJ, Hallett M, Louis ED, Raethjen J, Stamelou M, Testa CM, Deuschl G, Tremor Task Force of the International Parkinson and Movement Disorder Society (2018) Consensus Statement on the Classification of Tremors from the Task Force on Tremor of the International Parkinson and Movement Disorder Society. Mov Disord 33:75-87.

Bologna M, Paparella G, Fabbrini A, Leodori G, Rocchi L, Hallett M, Berardelli A (2016) Effects of cerebellar theta-burst stimulation on arm and neck movement kinematics in patients with focal dystonia. Clin Neurophysiol 127:3472-3479.

Bove F, Di Lazzaro G, Mulas D, Cocciolillo F, Di Giuda D, Bentivoglio AR (2018) A role for accelerometry in the differential diagnosis of tremor syndromes. Funct Neurol 33:45-49.

Brighina F, Romano M, Giglia G, Saia V, Puma A, Giglia F, Fierro B (2009) Effects of cerebellar TMS on motor cortex of patients with focal dystonia: a preliminary report. Exp Brain Res 192:651-656.

Brittain JS, Cagnan H, Mehta AR, Saifee TA, Edwards MJ, Brown P (2015) Distinguishing the central drive to tremor in Parkinson's disease and essential tremor. J Neurosci 35:795-806.

Cerasa A, Nistico R, Salsone M, Bono F, Salvino D, Morelli M, Arabia G, Quattrone A (2014) Neuroanatomical correlates of dystonic tremor: a cross-sectional study. Parkinsonism Relat Disord 20:314-317.

Conte A, Ferrazzano G, Belvisi D, Manzo N, Battista E, Li Voti P, Nardella A, Fabbrini G, Berardelli A (2018) Somatosensory temporal discrimination in Parkinson's disease, dystonia and essential tremor: pathophysiological and clinical implications. Clin Neurophysiol 129:1849-1853.

Cury RG, Fraix V, Castrioto A, Pérez Fernández MA, Krack P, Chabardes S, Seigneuret E, Alho EJ, Benabid AL, Moro E (2017) Thalamic deep brain stimulation for tremor in Parkinson disease, essential tremor, and dystonia. Neurology 89:1416-1423.

Daskalakis ZJ, Paradiso GO, Christensen BK, Fitzgerald PB, Gunraj C, Chen R (2004) Exploring the connectivity between the cerebellum and motor cortex in humans. J Physiol 557:689-700.

Defazio G, Conte A, Gigante AF, Fabbrini G, Berardelli A (2015) Is tremor in dystonia a phenotypic feature of dystonia? Neurology 84:1053-1059.

Defazio G, Gigante AF, Abbruzzese G, Bentivoglio AR, Colosimo C, Esposito M, Fabbrini G, Guidubaldi A, Girlanda P, Liguori R, Marinelli L, Morgante F, Santoro L, Tinazzi M, Livrea P, Berardelli A (2013) Tremor in primary adult-onset dystonia: prevalence and associated clinical features. J Neurol Neurosurg Psychiatry 84:404-408.

DeSimone JC, Archer DB, Vaillancourt DE, Wagle Shukla A (2019) Network-level connectivity is a critical feature distinguishing dystonic tremor and essential tremor. Brain 142:1644-1659. 
Deuschl G, Bain P, Brin M (1998) Consensus statement of the Movement Disorder Society on Tremor Ad Hoc Scientific Committee. Mov Disord 13 Suppl 3:2-23.

Deuschl G, Raethjen J, Lindemann M, Krack P (2001) The pathophysiology of tremor. Muscle Nerve 24:716-735.

di Biase L, Brittain JS, Shah SA, Pedrosa DJ, Cagnan H, Mathy A, Chen CC, Martín-Rodríguez JF, Mir P, Timmerman L, Schwingenschuh P, Bhatia K, Di Lazzaro V, Brown P (2017) Tremor stability index: a new tool for differential diagnosis in tremor syndromes. Brain 140:1977-1986.

Dirkx MF, den Ouden H, Aarts E, Timmer M, Bloem BR, Toni I, Helmich RC (2016) The cerebral network of Parkinson's tremor: an effective connectivity fMRI study. J Neurosci 36:5362-5372.

Farmer SF, Bremner FD, Halliday DM, Rosenberg JR, Stephens JA (1993) The frequency content of common synaptic inputs to motoneurones studied during voluntary isometric contraction in man. J Physiol 470:127-155.

Fisher KM, Lai HM, Baker MR, Baker SN (2009) Corticospinal activation confounds cerebellar effects of posterior fossa stimuli. Clin Neurophysiol 120:2109-2113.

Gallea C, Herath P, Voon V, Lerner A, Ostuni J, Saad Z, Thada S, Solomon J, Horovitz SG, Hallett M (2018) Loss of inhibition in sensorimotor networks in focal hand dystonia. Neuroimage Clin 17:90-97.

Grimaldi G, Argyropoulos GP, Boehringer A, Celnik P, Edwards MJ, Ferrucci R, Galea JM, Groiss SJ, Hiraoka K, Kassavetis P, Lesage E, Manto M, Miall RC, Priori A, Sadnicka A, Ugawa Y, Ziemann U (2014) Non-invasive cerebellar stimulation: a consensus paper. Cerebellum 13:121-138.

Hallett M (2011) Neurophysiology of dystonia: the role of inhibition. Neurobiol Dis 42:177-184.

Halliday DM, Rosenberg JR, Amjad AM, Breeze P, Conway BA, Farmer SF (1995) A framework for the analysis of mixed time series/point process data: theory and application to the study of physiological tremor, single motor unit discharges and electromyograms. Prog Biophys Mol Biol 64:237-278.

Hanajima R, Tsutsumi R, Shirota Y, Shimizu T, Tanaka N, Ugawa Y (2016) Cerebellar dysfunction in essential tremor. Mov Disord 31:1230-1234.

Helmich RC, Hallett M, Deuschl G, Toni I, Bloem BR (2012) Cerebral causes and consequences of parkinsonian resting tremor: a tale of two circuits? Brain 135:3206-3226.

Iwata NK, Ugawa Y (2005) The effects of cerebellar stimulation on the motor cortical excitability in neurological disorders: a review. Cerebellum $4: 218-223$.

Jedynak CP, Bonnet AM, Agid Y (1991) Tremor and idiopathic dystonia. Mov Disord 6:230-236.

Kassavetis P, Hoffland BS, Saifee TA, Bhatia KP, van de Warrenburg BP, Rothwell JC, Edwards MJ (2011) Cerebellar brain inhibition is decreased in active and surround muscles at the onset of voluntary movement. Exp Brain Res 209:437-442.

Kirke DN, Battistella G, Kumar V, Rubien-Thomas E, Choy M, Rumbach A, Simonyan K (2017) Neural correlates of dystonic tremor: a multimodal study of voice tremor in spasmodic dysphonia. Brain Imaging Behav 11:166-175.

Koch G, Porcacchia P, Ponzo V, Carrillo F, Cáceres-Redondo MT, Brusa L, Desiato MT, Arciprete F, et al. (2014) Effects of two weeks of cerebellar theta burst stimulation in cervical dystonia patients. Brain Stimul 7:564572 .

Louis ED (2019) Essential tremor: a nuanced approach to the clinical features. Pract Neurol 19:389-398.

Ma K, Babij R, Cortés E, Vonsattel JP, Louis ED (2012) Cerebellar pathology of a dual clinical diagnosis: patients with essential tremor and dystonia. Tremor Other Hyperkinet Mov (NY) 2:12.

Merola A, Dwivedi AK, Shaikh AG, Tareen TK, Da Prat GA, Kauffman MA, Hampf J, Mahajan A, Marsili L, Jankovic J, Comella CL, Berman BD, Perlmutter JS, Jinnah HA, Espay AJ (2019) Head tremor at disease onset: an ataxic phenotype of cervical dystonia. J Neurol 266:1844-1851.
Molnar GF, Sailer A, Gunraj CA, Lang AE, Lozano AM, Chen R (2004) Thalamic deep brain stimulation activates the cerebellothalamocortical pathway. Neurology 63:907-909.

Munchau A, Schrag A, Chuang C, MacKinnon CD, Bhatia KP, Quinn NP, Rothwell JC (2001) Arm tremor in cervical dystonia differs from essential tremor and can be classified by onset age and spread of symptoms. Brain 124:1765-1776.

Nieuwhof F, Panyakaew P, van de Warrenburg BP, Gallea C, Helmich RC (2018) The patchy tremor landscape: recent advances in pathophysiology. Curr Opin Neurol 31:455-461.

Nishimura Y, Morichika Y, Isa T (2009) A subcortical oscillatory network contributes to recovery of hand dexterity after spinal cord injury. Brain 132:709-721.

Nistico R, Pirritano D, Salsone M, Valentino P, Novellino F, Condino F, Bono F, Quattrone A (2012) Blink reflex recovery cycle in patients with dystonic tremor: a cross-sectional study. Neurology 78:1363-1365.

Norton JA, Wood DE, Marsden JF, Day BL (2003) Spinally generated electromyographic oscillations and spasms in a low-thoracic complete paraplegic. Mov Disord 18:101-106.

Ondo W, Hashem V, LeWitt PA, Pahwa R, Shih L, Tarsy D, Zesiewicz T, Elble R (2018) Comparison of the Fahn-Tolosa-Marin Clinical Rating Scale and the Essential Tremor Rating Assessment Scale. Mov Disord Clin Pract 5:60-65.

Pandey S, Sarma N (2016) Tremor in dystonia. Parkinsonism Relat Disord 29:3-9.

Panyakaew P, Cho HJ, Srivanitchapoom P, Popa T, Wu T, Hallett M (2016) Cerebellar brain inhibition in the target and surround muscles during voluntary tonic activation. Eur J Neurosci 43:1075-1081.

Pinto AD, Lang AE, Chen R (2003) The cerebellothalamocortical pathway in essential tremor. Neurology 60:1985-1987.

Porcacchia P, Álvarez de Toledo P, Rodríguez-Baena A, Martín-Rodríguez JF, Palomar FJ, Vargas-González L, Jesús S, Koch G, Mir P (2019) Abnormal cerebellar connectivity and plasticity in isolated cervical dystonia. PLoS One 14:e211367.

Quartarone A, Ruge D (2018) How many types of dystonia? Pathophysiological considerations. Front Neurol 9:12.

Rudzińska M, Krawczyk M, Wójcik-Peôdziwiatr M, Szczudlik A, Wasielewska A (2013) Tremor associated with focal and segmental dystonia. Neurol Neurochir Pol 47:223-231.

Schiebler S, Schmidt A, Zittel S, Baumer T, Gerloff C, Klein C, Munchau A (2011) Arm tremor in cervical dystonia: is it a manifestation of dystonia or essential tremor? Mov Disord 26:1789-1792.

Schwingenschuh P, Katschnig P, Seiler S, Saifee TA, Aguirregomozcorta M, Cordivari C, Schmidt R, Rothwell JC, Bhatia KP, Edwards MJ (2011) Moving toward "laboratory-supported" criteria for psychogenic tremor. Mov Disord 26:2509-2515.

Shaikh AG, Jinnah HA, Tripp RM, Optican LM, Ramat S, Lenz FA, Zee DS (2008) Irregularity distinguishes limb tremor in cervical dystonia from essential tremor. J Neurol Neurosurg Psychiatry 79:187-189.

Shaikh AG, Zee DS, Jinnah HA (2015) Oscillatory head movements in cervical dystonia: dystonia, tremor, or both? Mov Disord 30:834-842.

Shakkottai VG, Batla A, Bhatia K, Dauer WT, Dresel C, Niethammer M, Eidelberg D, Raike RS, Smith Y, Jinnah HA, Hess EJ, Meunier S, Hallett M, Fremont R, Khodakhah K, LeDoux MS, Popa T, Gallea C, Lehericy S, Bostan AC, et al. (2017) Current opinions and areas of consensus on the role of the cerebellum in dystonia. Cerebellum 16:577-594.

Tinazzi M, Fasano A, Di Matteo A, Conte A, Bove F, Bovi T, Peretti A, Defazio G, Fiorio M, Berardelli A (2013) Temporal discrimination in patients with dystonia and tremor and patients with essential tremor. Neurology 80:76-84.

Ugawa Y (2009) Can we see the cerebellar activation effect by TMS over the back of the head? Clin Neurophysiol 120:2006-2007.

Ugawa Y, Uesaka Y, Terao Y, Hanajima R, Kanazawa I (1995) Magnetic stimulation over the cerebellum in humans. Ann Neurol 37:703-713.

Ziemann U (2004) TMS and drugs. Clin Neurophysiol 115:1717-1729. 